\title{
85734 - CAUSAS BÁSICAS DE ÓBITO EM LONGEVOS DIABÉTICOS NO RS: DADOS DO DATASUS 2017
}

\author{
Pôster - Gerontologia
}

\author{
Vivian Ulrich / Ulrich, V / PUCRS; Renata Breda Martins / Martins, RB / PUCRS; \\ Luana de Souza Goulart / Goulart, LS / PUCRS; Ângelo José Gonçalves Bós / Bós, AJG / PUCRS
}

Introdução: Idosos diabéticos apresentam risco elevado de morte prematura e elevada predisposição a comorbidades ${ }^{1}$, sendo os longevos ( 80 anos ou mais) os mais vulneráveis. As causas de óbitos são consideradas umas das informações de maior relevância para o conhecimento da situação de saúde de uma população, subsidiando o planejamento e a administração de programas $^{2}$. A causa básica revela a doença inicial da sequência que levou ao falecimento e, portanto, ela deve orientar as ações em saúde no sentido de sua prevenção ${ }^{3}$. Objetivos: Investigar as principais causas básicas de óbito em longevos com diabetes mellitus tipo II. Métodos: Foram analisados os dados do Sistema de Informação de Mortalidade (SIM) do Departamento de Informática do SUS (DATASUS) referentes às principais causas de óbito em longevos do Rio Grande do Sul. Foi utilizado os capítulos "E10-E14 - diabetes" da Classificação Internacional de Doenças versão 10 (CID 10) para identificar os diabéticos. Os dados foram analisados com o programa Epi Info versão 7.2. Resultados: Dos 27.205 longevos cadastrados, 9,6\% $(\mathrm{n}=2.573)$ eram diabéticos. Observou-se maior frequência de diabetes em mulheres $(66,3 \% \mathrm{n}=1.706)$, indivíduos com 4 anos ou mais de estudo $(40,0 \% \mathrm{n}=929)$, brancos $(92,2 \% \mathrm{n}=2.289)$ e viúvos $57,2 \%(\mathrm{n}=1.404)$. Dentre as quatro principais causas básicas de mortes em longevos diabéticos estão: a própria diabetes (50,4\% $\mathrm{n}=1.295)$; doenças do aparelho circulatório $(22,7 \% \mathrm{n}=583)$; doenças do aparelho respiratório $(10,0 \% \mathrm{n}=256)$; neoplasias $(6,3 \% \mathrm{n}=162)$; e outras causas $(10,6 \% \mathrm{n}=276)$. Conclusões: Os dados levam a concluir que os longevos diabéticos não estão recebendo tratamento adequado.

Palavras-chave: Diabetes Mellitus. Multimorbidade. Idosos de 80 Anos ou mais.

Referências: 1. Sociedade Brasileira de Diabetes. Diretrizes da Sociedade Brasileira de Diabetes 2017-2018. Organização José Egídio Paulo de Oliveira, Renan Magalhães Montenegro Júnior, Sérgio Vencio. - São Paulo: Editora Clannad, 2017; 2. Silva VL, Albuquerque MFPM, Cesse EAP, Luna CF. Perfil de mortalidade do idoso: análise da evolução temporal em uma capital do Nordeste brasileiro de 1996 a 2007. Revista Brasileira Geriatria e Gerontologia. 2012;153:433-41; 3. Ishitani LH, França E. Uso das causas múltiplas de morte em saúde pública. Inf Epidemiol SUS. 2001;104:163-75. 